\title{
Radical structures of intuitionistic fuzzy polynomial ideals of a ring
}

\author{
P. K. Sharma ${ }^{1}$ and Gagandeep Kaur ${ }^{2}$ \\ ${ }^{1}$ Post Graduate Department of Mathematics, D.A.V. College \\ Jalandhar, Punjab, India \\ e-mail: pksharma@davjalandhar.com \\ ${ }^{2}$ Research Scholar, IKG PT University \\ Jalandhar, Punjab, India \\ e-mail: taktogagan@gmail.com
}

Received: 12 January $2018 \quad$ Revised: 7 November $2018 \quad$ Accepted: 11 November 2018

\begin{abstract}
In this paper we investigate the radical structure of an intuitionistic fuzzy polynomial ideal $A_{x}$ induced by an intuitionistic fuzzy ideal $A$ of a ring and study its properties. Given an intuitionistic fuzzy ideal $B$ of a ring $R^{\prime}$ and a homomorphism $f: R \rightarrow R^{\prime}$, we show that if $f_{x}: R[x] \rightarrow R^{\prime}[x]$ is the induced homomorphism of $f$, that is, $f_{x}\left(\sum_{i=0}^{n} a_{i} x^{i}\right)=\sum_{i=0}^{n}\left(f\left(a_{i}\right)\right) x^{i}$, then $f_{x}^{-1}\left[(\sqrt{B})_{x}\right]=\left(\sqrt{f^{-1}(B)}\right)_{x}$.
\end{abstract}

Keywords: Intuitionistic fuzzy polynomial ideal, Intuitionistic fuzzy ideal, $f$-invariant, Intuitionistic fuzzy prime (maximal) ideal.

2010 Mathematics Subject Classification: 03E72, 03F55, 13 F20.

\section{Introduction}

One of the remarkable generalizations of the fuzzy sets [14] is the intuitionistic fuzzy sets which was introduced by Atanassov [1,2]. Biswas was the first one to introduce the intuitionistic fuzzification of the algebraic structure and developed the concept of intuitionistic fuzzy subgroup of a group in [5]. Later on Hur and others in [7] and [6] defined and studied intuitionistic fuzzy subrings and ideals of a ring. With a different approach Banerjee and Basnet in [4] also studied intuitionistic fuzzy subrings and ideals of a ring. Jun and other in [8] introduced and study the 
notion of intuitionistic nil radicals of intuitionistic fuzzy ideals and Euclidean intuitionistic fuzzy ideals in rings. Translate of intuitionistic fuzzy subring and ideal was studied by Sharma in [12]. Meena and Thomas in [11] studied the concept of intuitionistic fuzzy subring to lattice setting and introduced the notion of intuitionistic L-fuzzy subring. The concept of Characteristic intuitionistic fuzzy subrings of an intuitionistic fuzzy ring was introduced by Meena in [10]. The present authors [13] introduced the notion of an intuitionistic fuzzy polynomial ideal $A_{x}$ of a polynomial ring $R[x]$ induced by an intuitionistic fuzzy ideal $A$ of a ring $\mathrm{R}$ and obtained an isomorphism theorem of a ring of an intuitionistic fuzzy cosets of $A_{x}$. It was shown that an intuitionistic fuzzy ideal $A$ of a ring is an intuitionistic fuzzy prime if and only if $A_{x}$ is an intuitionistic fuzzy prime ideal of $R[x]$. Moreover, it was shown that if $A_{x}$ is an intuitionistic fuzzy maximal ideal of $R[x]$, then $A$ is an intuitionistic fuzzy ideal of $R^{\prime}$.

In this paper, we investigate the radical structure of intuitionistic fuzzy polynomial ideal induced by an intuitionistic fuzzy ideal of a ring and study its properties.

\section{Preliminaries}

Definition 2.1. ([1]) Let $X$ be a non-empty fixed set. An intuitionistic fuzzy set (IFS) $A$ in $X$ is an object having the form $A=\left\{\left\langle x, \mu_{A}(x), \nu_{A}(x)\right\rangle \mid x \in X\right\}$, where the functions $\mu_{A}: X \rightarrow[0,1]$ and $\nu_{A}: X \rightarrow[0,1]$ denote the degree of membership (namely $\mu_{A}(x)$ ) and the degree of non-membership (namely $\nu_{A}(x)$ ) of each element $x \in X$ to the set $A$ respectively and $\mu_{A}(x)+\nu_{A}(x) \leq 1$ for each $x \in X$.

Remark 2.2. (i) When $\mu_{A}(x)+\nu_{A}(x)=1, \forall x \in X$. Then $A$ is called a fuzzy set.

(ii) We denote the IFS $A=\left\{\left\langle x, \mu_{A}(x), \nu_{A}(x)\right\rangle \mid x \in X\right\}$ by $A=\left(\mu_{A}, \nu_{A}\right)$.

Definition 2.3. ([2,11]) Let $A=\left\{\left\langle x, \mu_{A}(x), \nu_{A}(x)\right\rangle \mid x \in X\right\}$ and $B=\left\{\left\langle x, \mu_{B}(x), \nu_{B}(x)\right\rangle \mid x \in\right.$ $X\}$ be any two IFSs of $X$, then

(i) $A \subseteq B$ if and only if $\mu_{A}(x) \leq \mu_{B}(x)$ and $\nu_{A}(x) \geq \nu_{B}(x)$ for all $x \in X$

(ii) $A=B$ if and only if $\mu_{A}(x)=\mu_{B}(x)$ and $\nu_{A}(x)=\nu_{B}(x)$ for all $x \in X$

(iii) $A^{c}=\left\{\left\langle x, \mu_{A^{c}}(x), \nu_{A^{c}}(x)\right\rangle \mid x \in X\right\}$, where $\mu_{A^{c}}(x)=\nu_{A}(x)$ and $\nu_{A^{c}}(x)=\mu_{A}(x)$ for all $x \in X$

(iv) $A \cap B=\left\{\left\langle x, \mu_{A \cap B}(x), \nu_{A \cap B}(x)\right\rangle \mid x \in X\right\}$, where $\mu_{A \cap B}(x)=\mu_{A}(x) \wedge \mu_{B}(x)$ and $\nu_{A \cap B}(x)=$ $\nu_{A}(x) \vee \nu_{B}(x)$

(v) $A \cup B=\left\{\left\langle x, \mu_{A \cup B}(x), \nu_{A \cup B}(x)\right\rangle \mid x \in X\right\}$, where $\mu_{A \cup B}(x)=\mu_{A}(x) \vee \mu_{B}(x)$ and $\nu_{A \cup B}(x)=$ $\nu_{A}(x) \wedge \nu_{B}(x)$.

Definition 2.4. ([3, 4, 6, 11]) Let $R$ be a ring. An IFS $A=\left(\mu_{A}, \nu_{A}\right)$ of $R$ is said to be an intuitionistic fuzzy ideal (IFI) of $R$ if

(i) $\mu_{A}(x-y) \geq \mu_{A}(x) \wedge \mu_{A}(y)$ and $\nu_{A}(x-y) \leq \nu_{A}(x) \vee \nu_{A}(y)$;

(ii) $\mu_{A}(x y) \geq \mu_{A}(x) \vee \mu_{A}(y)$ and $\nu_{A}(x y) \leq \nu_{A}(x) \wedge \nu_{A}(y), \forall x, y \in R$. 
If $A$ is an intuitionistic fuzzy ideal of $R$, then

(a) $\mu_{A}(0) \geq \mu_{A}(x) \geq \mu_{A}(1)$ and $\nu_{A}(0) \leq \nu_{A}(x) \leq \nu_{A}(1), \forall x \in R$.

(b) $\mu_{A}(x-y)=\mu_{A}(0)$ and $\nu_{A}(x-y)=\nu_{A}(0) \Leftrightarrow \mu_{A}(x)=\mu_{A}(y)$ and $\nu_{A}(x)=\nu_{A}(y)$.

(c) The $(\alpha, \beta)$-cut set of $A$, i.e., the set $C_{(\alpha, \beta)}(A)=\left\{x \in R \mid \mu_{A}(x) \geq \alpha\right.$ and $\left.\nu_{A}(x) \leq \beta\right\}$ is an ideal of $R$, where $\alpha, \beta \in[0,1]$ such that $\alpha+\beta \leq 1$.

(d) If $A$ and $B$ are two IFIs of the ring $R$, then sum $A+B$ and the product $A B$ are defined as: $\mu_{A+B}(x)=\vee_{x=y+z}\left\{\mu_{A}(y) \wedge \mu_{B}(z)\right\}$ and $\nu_{A+B}(x)=\wedge_{x=y+z}\left\{\nu_{A}(y) \vee \nu_{B}(z)\right\}, \forall x \in R$ and $\mu_{A B}(x)=\vee_{x=y z}\left\{\mu_{A}(y) \wedge \mu_{B}(z)\right\}$ and $\nu_{A B}(x)=\wedge_{x=y z}\left\{\nu_{A}(y) \vee \nu_{B}(z)\right\}, \forall x \in R$.

Definition 2.5. ([3,4]) Let $f: R \rightarrow S$ be a homomorphism of rings and B be an IFS of $S$. We define an IFS $f^{-1}(B)$ of $R$ by $f^{-1}(B)(x):=B(f(x)), \forall x \in R$.

Definition 2.6. ([3, 4]) Let $f: R \rightarrow S$ be a homomorphism of rings and A be an IFS of $R$. We define an IFS $f(A)$ of $S$ by $f(A)(y)=\left(\mu_{f(A)}(y), \nu_{f(A)}(y)\right)$, where $\forall y \in S$,

$$
f(A)(y)= \begin{cases}\left(\vee\left\{\mu_{A}(x) \mid x \in f^{-1}(y)\right\}, \wedge\left\{\nu_{A}(x) \mid x \in f^{-1}(y)\right\}\right), & \text { if } f^{-1}(y) \neq \phi \\ (0,1), & \text { otherwise }\end{cases}
$$

Definition 2.7. ([8]) Let $R$ and $S$ be any sets and let $f: R \rightarrow S$ be a function. An IFS $A$ of $R$ is called an $f$-invariant if $f(x)=f(y) \Rightarrow \mu_{A}(x)=\mu_{A}(y)$ and $\nu_{A}(x)=\nu_{A}(y)$, where $x, y \in R$. If $A$ is any $f$-invariant IFS of $R$, then $f^{-1}(f(A))=A$.

Definition 2.8. ([9]) Let $f: R \rightarrow R^{\prime}$ be a homomorphism of rings. A map $f_{x}: R[x] \rightarrow R^{\prime}[x]$ defined by

$$
f_{x}\left(\sum_{i=0}^{n} a_{i} x^{i}\right)=\sum_{i=0}^{n} f\left(a_{i}\right) x^{i}
$$

is obviously a ring homomorphism, and we call $f_{x}$ an induced homomorphism by $f$.

Theorem 2.9. ([13]) Let $A=\left(\mu_{A}, \nu_{A}\right)$ be an IFI of a ring $R$ and let $f(x)=\sum_{i=0}^{n} a_{i} x^{i} \in R[x]$. Define an IFS $A_{x}=\left(\mu_{A_{x}}, \nu_{A_{x}}\right)$ on $R[x]$ by

$$
\mu_{A_{x}}(f(x))=\min _{i}\left\{\mu_{A}\left(a_{i}\right)\right\} \text { and } \nu_{A_{x}}(f(x))=\max _{i}\left\{\nu_{A}\left(a_{i}\right)\right\} .
$$

Then $A_{x}$ is an IFI of $R[x]$.

The intuitionistic fuzzy ideal $A_{x}$ is called the intuitionistic fuzzy polynomial ideal of $R[x]$ induced by an intuitionistic fuzzy ideal $A$ of $R$.

Proposition 2.10. ([13]) Let $f: R \rightarrow R^{\prime}$ be a homomorphism of rings and let $f_{x}: R[x] \rightarrow R^{\prime}[x]$ be an induced homomorphism of $f$. If $A$ is an IFI of the ring $R$ and $A_{x}$ be its the intuitionistic fuzzy polynomial ideal of $R[x]$, then $A$ is $f$-invariant if and only if $A_{x}$ is $f_{x}$-invariant.

Proposition 2.11. ([13]) Let $A$ be an IFI of the ring $R$. Then the set $S=\left\{f(x) \in R[x] \mid \mu_{A_{x}}(f(x))=\mu_{A_{x}}(0)\right.$ and $\left.\nu_{A_{x}}(f(x))=\nu_{A_{x}}(0)\right\}$ is a subring of $R[x]$. 
Remark 2.12. ([11]) Let $A$ be an IFS of a ring $R$. We denote a level cut set $A_{*}$ by

$$
A_{*}=\left\{x \in R \mid \mu_{A}(x)=\mu_{A}(0) \text { and } \nu_{A}(x)=\nu_{A}(0)\right\} .
$$

It is proved in [11] that if $A$ is an IFI of ring $R$, then $A_{*}$ is an ideal of ring $R$. Note that if $A$ is an IFI of a ring $R$, then $\mu_{A}(0) \geq \mu_{A}(x)$ and $\nu_{A}(0) \leq \nu_{A}(x)$ for all $x \in R$.

We denote $A_{*}[x]=\left\{f(x)=\sum_{i=0}^{n} a_{i} x^{i} \in R[x] \mid a_{i} \in A_{*}, \forall i=1,2, \ldots, n\right\}$.

Theorem 2.13. ([13]) Let $A$ be an IFI of a ring $R$, then $\left(A_{x}\right)_{*}=A_{*}[x]$.

Theorem 2.14. ([13]) If $A$ and $B$ are two IFIs of a ring $R$, then

(i) $(A \cap B)_{x}=A_{x} \cap B_{x}$.

(ii) $(A \cup B)_{x} \supseteq A_{x} \cup B_{x}$.

(iii) $A_{x}+B_{x} \subseteq(A+B)_{x}$.

(iv) $A_{x} B_{x} \subseteq(A B)_{x}$.

Theorem 2.15. ([13]) Let $f: R \rightarrow R^{\prime}$ be a homomorphism from $R$ onto $R^{\prime}$ and let $f_{x}$ be an induced homomorphism of $f$. If $A$ is an $f$-invariant IFIs of $R^{\prime}$, then $(f(A))_{x}=f_{x}\left(A_{x}\right)$.

Theorem 2.16. ([13]) Let $A$ be an IFI of a ring $R$. Then $A$ is an intuitionistic fuzzy prime ideal of $R$ if and only if $A_{x}$ is an intuitionistic fuzzy prime ideal of $R[x]$.

\section{Radical of the intuitionistic fuzzy polynomial induced by an intuitionistic fuzzy ideal}

In this section, we study some relations between the radical of the intuitionistic fuzzy polynomial ideal $R[x]$ induced by an intuitionistic fuzzy ideal of a ring $R$ and the radical of an intuitionistic fuzzy ideal of the ring.

Definition 3.1. ([8]) Let $A=\left(\mu_{A}, \nu_{A}\right)$ be an intuitionistic fuzzy ideal of $R$. Then the intuitionistic fuzzy nil radical of $A$ is defined to be an IFS $\sqrt{A}=\left(\mu_{\sqrt{A}}, \nu_{\sqrt{A}}\right)$ defined by $\mu_{A}(x)=$ $\vee\left\{\mu_{A}\left(x^{n}\right) \mid n>0\right\}$ and $\nu_{A}(x)=\vee\left\{\nu_{A}\left(x^{n}\right) \mid n>0\right\}, \forall x \in R$ and for some $n \in \mathbf{N}$.

Proposition 3.2. ([8]) For any intuitionistic fuzzy ideals $A$ and $B$ of $R$, we have

(i) $A \subseteq \sqrt{A}$

(ii) If $A \subseteq B$ then $\sqrt{A} \subseteq \sqrt{B}$

(iii) $\sqrt{\sqrt{A}}=\sqrt{A}$.

Proof. Straightforward.

Theorem 3.3. For any intuitionistic fuzzy ideals $A$ of $R, \sqrt{A}$ is an intuitionistic fuzzy ideal of $R$.

Proof. Let $x, y \in R$. Then 


$$
\begin{aligned}
\mu_{\sqrt{A}}(x) \wedge \mu_{\sqrt{A}}(y) & =\left(\vee\left\{\mu_{A}\left(x^{m}\right) \mid m>0\right\}\right) \wedge\left(\vee\left\{\mu_{A}\left(y^{n}\right) \mid n>0\right\}\right) \\
& =\vee\left\{\left(\vee\left\{\mu_{A}\left(x^{m}\right) \wedge \mu_{A}\left(y^{n}\right) \mid n>0\right\}\right) \mid m>0\right\} .
\end{aligned}
$$

Thus,

$$
\mu_{\sqrt{A}}(x) \wedge \mu_{\sqrt{A}}(y)=\vee\left\{\left(\vee\left\{\mu_{A}\left(x^{m}\right) \wedge \mu_{A}\left(y^{n}\right) \mid n>0\right\}\right) \mid m>0\right\}
$$

Similarly,

$$
\nu_{\sqrt{A}}(x) \vee \nu_{\sqrt{A}}(y)=\wedge\left\{\left(\wedge\left\{\nu_{A}\left(x^{m}\right) \vee \nu_{A}\left(y^{n}\right) \mid n>0\right\}\right) \mid m>0\right\} .
$$

Let $m$ and $n$ be any positive integers. Since $R$ is commutative, we know that each term in the binomial expansion of $(x+y)^{m+n}$ contains either $x^{m}$ or $y^{n}$ as a factor. Hence there exist $r, t \in R$ such that $(x+y)^{m+n}=r x^{m}+t y^{n}$. Thus

$$
\begin{aligned}
\mu_{A}\left(x^{m}\right) \wedge \mu_{A}\left(y^{n}\right) & \leq\left(\mu_{A}\left(x^{m}\right) \vee \mu_{A}(r)\right) \wedge\left(\mu_{A}\left(y^{n}\right) \vee \mu_{A}(t)\right) \\
& \leq \mu_{A}\left(r x^{m}\right) \wedge \mu_{A}\left(t y^{n}\right) \\
& \leq \mu_{A}\left(r x^{m}+t y^{n}\right) \\
& =\mu_{A}\left((x+y)^{m+n}\right) \\
& \leq \vee\left\{\mu_{A}\left((x+y)^{k}\right) \mid k>0\right\} \\
& =\mu_{\sqrt{A}}(x+y) .
\end{aligned}
$$

Thus,

$$
\mu_{A}\left(x^{m}\right) \wedge \mu_{A}\left(y^{n}\right) \leq \mu_{\sqrt{A}}(x+y)
$$

Similarly,

$$
\nu_{A}\left(x^{m}\right) \vee \nu_{A}\left(y^{n}\right) \geq \nu_{\sqrt{A}}(x+y) .
$$

Notice that $\mu_{A}(x-y) \geq \mu_{A}(x) \wedge \mu_{A}(y) \Leftrightarrow \mu_{A}(x+y) \geq \mu_{A}(x) \wedge \mu_{A}(y)$ and $\nu_{A}(x-y) \leq$ $\nu_{A}(x) \vee \nu_{A}(y) \Leftrightarrow \nu_{A}(x+y) \leq \nu_{A}(x) \vee \nu_{A}(y)$, respectively. Next, we have

$$
\begin{aligned}
\mu_{\sqrt{A}}(x) \vee \mu_{\sqrt{A}}(y) & =\left(\vee\left\{\mu_{A}\left(x^{n}\right) \mid n>0\right\}\right) \vee\left(\vee\left\{\mu_{A}\left(y^{n}\right) \mid n>0\right\}\right) \\
& =\vee\left\{\left(\vee\left\{\mu_{A}\left(x^{n}\right) \vee \mu_{A}\left(y^{n}\right) \mid n>0\right\} .\right.\right.
\end{aligned}
$$

Thus,

$$
\mu_{\sqrt{A}}(x) \vee \mu_{\sqrt{A}}(y)=\vee\left\{\left(\vee\left\{\mu_{A}\left(x^{n}\right) \vee \mu_{A}\left(y^{n}\right)\right\}\right) \mid n>0\right\}
$$

Similarly,

$$
\nu_{\sqrt{A}}(x) \wedge \nu_{\sqrt{A}}(y)=\wedge\left\{\left(\nu_{A}\left(x^{n}\right) \wedge \nu_{A}\left(y^{n}\right)\right) \mid n>0\right\} .
$$

Since

$$
\begin{aligned}
\mu_{A}\left(x^{n}\right) \wedge \mu_{A}\left(y^{n}\right) & \leq \mu_{A}\left(x^{n} y^{n}\right) \\
& =\mu_{A}\left((x y)^{n}\right) \\
& \leq \vee\left\{\mu_{A}\left((x y)^{k}\right) \mid k>0\right\} \\
& =\mu_{\sqrt{A}}(x y) .
\end{aligned}
$$


Thus,

$$
\mu_{A}\left(x^{n}\right) \wedge \mu_{A}\left(y^{n}\right) \leq \mu_{\sqrt{A}}(x y) .
$$

From (3.5) and (3.6) we get $\mu_{\sqrt{A}}(x y) \geq \mu_{\sqrt{A}}(x) \wedge \mu_{\sqrt{A}}(y)$ and $\nu_{\sqrt{A}}(x y) \leq \nu_{\sqrt{A}}(x) \vee \nu_{\sqrt{A}}(y)$. Hence $\sqrt{A}$ is an intuitionistic fuzzy ideal of $R$.

Theorem 3.4. If $A$ and $B$ are intuitionistic fuzzy ideals of $R$, then

(i) $\sqrt{A \cap B}=\sqrt{A} \cap \sqrt{B}$

(ii) $\sqrt{A \cup B}=\sqrt{A} \cup \sqrt{B}$

(iii) $\sqrt{A}+\sqrt{B} \subseteq \sqrt{A+B}$.

(iv) $\sqrt{A} \sqrt{B} \subseteq \sqrt{A B}$.

Proof. (i) Since $A \cap B \subseteq A$ and $A \cap B \subseteq B$. Therefore, by Proposition (3.2)(i) we get $\sqrt{A \cap B} \subseteq$ $\sqrt{A}$ and $\sqrt{A \cap B} \subseteq \sqrt{B}$ and so, $\sqrt{A \cap B} \subseteq \sqrt{A} \cap \sqrt{B}$.

For another inclusion, let $x \in R$ be any element. Then

$$
\begin{aligned}
\mu_{\sqrt{A} \cap \sqrt{B}}(x) & =\mu_{\sqrt{A}}(x) \wedge \mu_{\sqrt{B}}(x) \\
& =\left(\vee\left\{\mu_{A}\left(x^{m}\right) \mid m>0\right\}\right) \wedge\left(\vee\left\{\mu_{B}\left(y^{n}\right) \mid n>0\right\}\right) \\
& =\vee\left\{\vee\left\{\mu_{A}\left(x^{m}\right) \wedge \mu_{B}\left(y^{n}\right) \mid n>0\right\} \mid m>0\right\} .
\end{aligned}
$$

Similarly, we can show $\nu_{\sqrt{A} \cap \sqrt{B}}(x)=\wedge\left\{\wedge\left\{\nu_{A}\left(x^{m}\right) \vee \nu_{B}\left(y^{n}\right) \mid n>0\right\} \mid m>0\right\}$.

Now, let $m$ and $n$ be any positive integers. Then,

$$
\begin{aligned}
\mu_{A}\left(x^{m}\right) \wedge \mu_{B}\left(y^{n}\right) & \leq \mu_{A}\left(x^{m n}\right) \wedge \mu_{B}\left(y^{m n}\right) \\
& =\mu_{A \cap B}\left(x^{m n}\right) \\
& \leq \vee\left\{\mu_{A \cap B}\left(x^{k}\right) \mid k>0\right\} \\
& =\mu_{\sqrt{A \cap B}}(x) .
\end{aligned}
$$

Thus, $\mu_{A}\left(x^{m}\right) \wedge \mu_{B}\left(y^{n}\right) \leq \mu_{\sqrt{A \cap B}}(x)$. Therefore, $\mu_{\sqrt{A} \cap \sqrt{B}}(x) \leq \mu_{\sqrt{A \cap B}}(x)$ Similarly, we can show that $\nu_{A}\left(x^{m}\right) \vee \nu_{B}\left(y^{n}\right) \geq \nu_{\sqrt{A \cap B}}(x)$ and so, $\nu_{\sqrt{A} \cap \sqrt{B}}(x) \geq \nu_{\sqrt{A \cap B}}(x)$. Hence $\sqrt{A} \cap \sqrt{B} \subseteq$ $\sqrt{A \cap B}$. This completes the proof of (i).

(ii) The proof follows similar to the proof of part (i).

(iii) The proof follows from the definition of sum of IFIs.

(iv) The proof follows from the definition of product of IFIs.

If $A$ is an intuitionistic fuzzy ideal of a ring $R$, then $A_{x}$ is an intuitionsitic fuzzy ideal of a polynomial ring $R[x]$ by Theorem 2.9, the IFS $\sqrt{A_{x}}$ is the intuitionistic fuzzy nil radical of $A_{x}$. The following theorem gives that the two intuitionistic fuzzy nil radicals have the same value.

Theorem 3.5. If $A$ is an intuitionistic fuzzy ideal of $R$, then $\left(\sqrt{A_{x}}\right)_{x}=(\sqrt{A})_{x}$.

Proof. Let $f(x)=\sum_{i=0}^{m} a_{i} x^{i} \in R[x]$ be any element of $R[x]$. Then by Theorem 2.9, we have $A_{x}\left(a_{j}^{n}\right)=\left(\mu_{A_{x}}\left(a_{j}^{n}\right), \nu_{A_{x}}\left(a_{j}^{n}\right)\right)$, where

$$
\mu_{A_{x}}\left(a_{j}^{n}\right)=\mu_{A_{x}\left(a_{j}^{n}+0 x+0 x^{2}+\ldots+0 x^{m}\right)}=\min \left\{\mu_{A}\left(a_{j}^{n}\right), \mu_{A}(0), \ldots, \mu_{A}(0)\right\}=\mu_{A}\left(a_{j}^{n}\right)
$$


and

$$
\nu_{A_{x}}\left(a_{j}^{n}\right)=\nu_{A_{x}}\left(a_{j}^{n}+0 x+0 x^{2}+\ldots+0 x^{m}\right)=\max \left\{\nu_{A}\left(a_{j}^{n}\right), \nu_{A}(0), \ldots, \nu_{A}(0)\right\}=\nu_{A}\left(a_{j}^{n}\right) .
$$

Since $\sqrt{A_{x}}$ is an intuitionistic fuzzy ideal of $R[x]$, we obtain

$$
\begin{aligned}
&\left(\sqrt{A_{x}}\right)_{x}(f(x))=\left(\mu_{\left(\sqrt{A_{x}}\right)_{x}}(f(x)), \nu_{\left(\sqrt{A_{x}}\right)_{x}}(f(x))\right), \text { where } \\
& \mu_{\left(\sqrt{A_{x}}\right)_{x}}(f(x))=\min _{i=0}\left\{\mu_{\sqrt{A_{x}}}\left(a_{i}\right)\right\} \\
&=\min _{i=0}^{m}\left\{\bigvee\left\{\mu_{A_{x}}\left(a_{i}^{n}\right) \mid n>0\right\}\right\} \\
&=\min _{i=0}\left\{\bigvee\left\{\mu_{A}\left(a_{i}^{n}\right) \mid n>0\right\}\right\}\left[\because \mu_{A_{x}}\left(a_{i}^{n}\right)=\mu_{A}\left(a_{i}^{n}\right)\right] \\
&=\min _{i=0}^{m}\left\{\mu_{\sqrt{A}}\left(a_{i}\right)\right\} \\
&=\mu_{(\sqrt{A})_{x}}(f(x)) .
\end{aligned}
$$

Similarly, we can show that $\nu_{\left(\sqrt{A_{x}}\right)_{x}}(f(x))=\nu_{(\sqrt{A})_{x}}(f(x))$.

This proves that $\left(\sqrt{A_{x}}\right)_{x}=(\sqrt{A})_{x}$.

Theorem 3.6. If $A$ and $B$ are intuitionistic fuzzy ideals of $R$, then

(i) $(\sqrt{A \cap B})_{x}=(\sqrt{A})_{x} \cap(\sqrt{B})_{x}$

(ii) $(\sqrt{A})_{x} \cup(\sqrt{B})_{x} \subseteq(\sqrt{A \cup B})_{x}$

(iii) $(\sqrt{A})_{x}+(\sqrt{B})_{x} \subseteq(\sqrt{A+B})_{x}$

(iv) $(\sqrt{A B})_{x} \subseteq(\sqrt{A})_{x}(\sqrt{B})_{x}$.

Proof. Let $A$ be an intuitionistic fuzzy ideal of $R$, then $A_{x}$ and $B_{x}$ are intuitionistic fuzzy polynomial ideals of $R[x]$ by Theorem 2.9.

For (i), we have

$$
\begin{aligned}
(\sqrt{A \cap B})_{x} & =\left(\sqrt{(A \cap B)_{x}}\right)_{x}[\text { Theorem 3.5] } \\
& =\left(\sqrt{A_{x} \cap B_{x}}\right)_{x}[\text { Theorem 2.14 (i) }] \\
& =\left(\sqrt{A_{x}} \cap \sqrt{B_{x}}\right)_{x}[\text { Lemma 3.1 (i)] } \\
& =\left(\sqrt{A_{x}}\right)_{x} \cap\left(\sqrt{B_{x}}\right)_{x}[\text { Theorem 2.14 (i) }] \\
& =(\sqrt{A})_{x} \cap(\sqrt{B})_{x}[\text { Theorem 3.5]. }
\end{aligned}
$$

For (ii), we have

$$
\begin{aligned}
(\sqrt{A})_{x} \cup(\sqrt{B})_{x} & =\left(\sqrt{A_{x}}\right)_{x} \cup\left(\sqrt{B_{x}}\right)_{x} \text { [Theorem 3.5] } \\
& \subseteq\left(\sqrt{A_{x}} \cup \sqrt{B_{x}}\right)_{x}[\text { Theorem 2.14 (ii)] } \\
& =\left(\sqrt{A_{x} \cup B_{x}}\right)_{x}[\text { Lemma 3.4 (ii)] } \\
& \subseteq\left(\sqrt{(A \cup B)_{x}}\right)_{x}[\text { Theorem 2.14 (ii)] } \\
& =(\sqrt{A \cup B})_{x}[\text { Theorem 3.5]. }
\end{aligned}
$$


For (iii), we have

$$
\begin{aligned}
(\sqrt{A})_{x}+(\sqrt{B})_{x} & =\left(\sqrt{A_{x}}\right)_{x}+\left(\sqrt{B_{x}}\right)_{x}[\text { Theorem 3.5] } \\
& \subseteq\left(\sqrt{A_{x}}+\sqrt{B_{x}}\right)_{x}[\text { Theorem 2.14 (iii)] } \\
& =\left(\sqrt{A_{x}+B_{x}}\right)_{x}[\text { Lemma 3.4 (iii) }] \\
& \subseteq\left(\sqrt{(A+B)_{x}}\right)_{x}[\text { Theorem 2.14 (iii) }] \\
& =(\sqrt{A+B})_{x}[\text { Theorem 3.5]. }
\end{aligned}
$$

For (iv), we have

$$
\begin{aligned}
(\sqrt{A})_{x}(\sqrt{B})_{x} & =\left(\sqrt{A_{x}}\right)_{x}\left(\sqrt{B_{x}}\right)_{x}[\text { Theorem 3.5] } \\
& \supseteq\left(\sqrt{A_{x}} \sqrt{B_{x}}\right)_{x}[\text { Theorem } 2.14 \text { (iv) }] \\
& =\left(\sqrt{A_{x} B_{x}}\right)_{x} \text { [Lemma } 3.4 \text { (iv)] } \\
& \supseteq\left(\sqrt{(A B)_{x}}\right)_{x} \text { [Theorem } 2.14 \text { (iv)] } \\
& =(\sqrt{A B})_{x}[\text { Theorem 3.5]. }
\end{aligned}
$$

This completes the proof.

Theorem 3.7. Let $B$ be an intuitionistic fuzzy ideal of $R^{\prime}$ and let $f: R \rightarrow R^{\prime}$ be a homomorphism of rings. If $f_{x}$ is the induced homomorphism of $f$, i.e., $f_{x}\left(\sum_{i=0}^{n} a_{i} x^{i}\right)=\sum_{i=0}^{n} f\left(a_{i}\right) x^{i}$, then $f_{x}^{-1}\left[(\sqrt{B})_{x}\right]=\left(\sqrt{f^{-1}(B)}\right)_{x}$.

Proof. Given a polynomial $g(x)=\sum_{i=0}^{m} b_{i} x^{i} \in R[x]$, we have

$$
\begin{aligned}
& \left(\sqrt{f^{-1}(B)}\right)_{x}(g(x))=\left(\mu_{\left(\sqrt{f^{-1}(B)}\right)_{x}}(g(x)), \nu_{\left(\sqrt{f^{-1}(B)}\right)_{x}}(g(x))\right), \text { where } \\
& \mu_{\left(\sqrt{f^{-1}(B)}\right)_{x}}(g(x))=\min _{i}^{m}\left\{\mu \sqrt{f^{-1}(B)}\left(b_{i}\right)\right\} \\
& =\min _{i}\left\{\vee\left\{\mu_{f^{-1}(B)}\left(b_{i}^{n}\right) \mid n>0\right\}\right\} \\
& =\vee\left\{\min _{i}^{m}\left\{\mu_{f^{-1}(B)}\left(b_{i}^{n}\right)\right\} \mid n>0\right\} \\
& =\vee\left\{\min _{i}^{m}\left\{\mu_{B}\left(f\left(b_{i}^{n}\right)\right)\right\} \mid n>0\right\} \\
& =\vee\left\{\min _{i}^{m}\left\{\mu_{B}\left(f\left(b_{i}\right)^{n}\right)\right\} \mid n>0\right\} \\
& =\min _{i}\left\{\vee\left\{\mu_{B}\left(f\left(b_{i}\right)^{n}\right) \mid n>0\right\}\right\} \\
& =\min _{i}\left\{\mu_{\sqrt{B}}\left(f\left(b_{i}\right)\right\}\right. \\
& =\mu_{(\sqrt{B})_{x}\left(f_{x}(g(x))\right)} \\
& =\mu_{f_{x}^{-1}\left((\sqrt{B})_{x}\right)}(g(x)) \text {. }
\end{aligned}
$$

Similarly, we can show that $\mu_{\left(\sqrt{f^{-1}(B)}\right)_{x}}(g(x))=\mu_{f_{x}^{-1}\left((\sqrt{B})_{x}\right)}(g(x))$.

Hence $f_{x}^{-1}\left[(\sqrt{B})_{x}\right]=\left(\sqrt{f^{-1}(B)}\right)_{x}$.

Proposition 3.8. Let $f: R \rightarrow R^{\prime}$ be an epimorphism from $R$ onto $R^{\prime}$ and let $A$ be an intuitionistic fuzzy ideal of $R$, then $f(\sqrt{A}) \subseteq \sqrt{f(A)}$. Further, if $A$ is constant on $\operatorname{Ker} f$, then $f(\sqrt{A})=$ $\sqrt{f(A)}$ 
Proof. Clearly, $f(A)$ and $f(\sqrt{A})$ are intuitionistic fuzzy ideals of $R^{\prime}$. If $y \in R^{\prime}$ and $f(x)=y$ for some $x \in R$, then $f\left(x^{n}\right)=y^{n}$, for all $n=1,2, \ldots$, then

$$
\begin{aligned}
\mu_{f(\sqrt{A})}(y) & =\sup \left\{\mu_{\sqrt{A}}(x) \mid x \in f^{-1}(y)\right\} \\
& =\sup \left\{\vee\left\{\mu_{A}\left(x^{n}\right) \mid n>0\right\} \mid x \in f^{-1}(y)\right\} \\
& =\vee\left\{\sup \left\{\mu_{A}\left(x^{n}\right) \mid x \in f^{-1}(y)\right\} \mid n>0\right\} \\
& \leq \vee\left\{\sup \left\{\mu_{A}\left(x^{n}\right) \mid x^{n} \in f^{-1}\left(y^{n}\right)\right\} \mid n>0\right\} \\
& =\vee\left\{\sup \left\{\mu_{A}\left(z^{n}\right) \mid z \in f^{-1}\left(y^{n}\right)\right\} \mid n>0\right\} \\
& =\vee\left\{\mu_{f(A)}\left(y^{n}\right) \mid n>0\right\} \\
& =\mu \sqrt{f(A)}(y) .
\end{aligned}
$$

Similarly, we can show that $\nu_{f(\sqrt{A})}(y) \geq \nu \sqrt{f(A)}(y)$. Thus, we have $f(\sqrt{A}) \subseteq \sqrt{f(A)}$.

Further, if $A$ is constant on $\operatorname{Ker} f$ and $x_{0} \in f^{-1}(y)$ is a fixed element, then by Proposition (2.3)(b) ensure that $\mu_{A}\left(x^{n}\right)=\mu_{A}\left(x_{0}^{n}\right)$ and $\nu_{A}\left(x^{n}\right)=\nu_{A}\left(x_{0}^{n}\right)$ for all $x \in f^{-1}(y)$ and $\mu_{A}(x)=$ $\mu_{A}\left(x_{0}^{n}\right)$ and $\nu_{A}(x)=\nu_{A}\left(x_{0}^{n}\right)$ for all $x \in f^{-1}\left(y^{n}\right)$. Hence

$$
\begin{aligned}
\mu_{f(\sqrt{A})}(y) & =\sup \left\{\mu_{\sqrt{A}}(x) \mid x \in f^{-1}(y)\right\} \\
& =\sup \left\{\vee\left\{\mu_{A}\left(x^{n}\right) \mid n>0\right\} \mid x \in f^{-1}(y)\right\} \\
& =\vee\left\{\sup \left\{\mu_{A}\left(x^{n}\right) \mid x \in f^{-1}(y)\right\} \mid n>0\right\} \\
& =\vee\left\{\sup \left\{\mu_{A}\left(x_{0}^{n}\right) \mid x \in f^{-1}(y)\right\} \mid n>0\right\} \\
& =\vee\left\{\sup \left\{\mu_{A}(x) \mid x \in f^{-1}\left(y^{n}\right)\right\} \mid n>0\right\} \\
& =\vee\left\{\mu_{f(A)}\left(y^{n}\right) \mid n>0\right\} \\
& =\mu \sqrt{f(A)}(y) .
\end{aligned}
$$

Similarly, we can show that $\mu_{f(\sqrt{A})}(y)=\mu \sqrt{f(A)}(y)$.

Thus, we have $f(\sqrt{A})=\sqrt{f(A)}$.

Theorem 3.9. Let $f: R \rightarrow R^{\prime}$ be a homomorphism from $R$ onto $R^{\prime}$ and let $f_{x}$ be the induced homomorphism of $f$. If an intuitionistic fuzzy ideal $A$ of $R$ is constant on $\operatorname{Ker} f$, then the intuitionistic fuzzy polynomial ideal $A_{x}$ is constant on $\operatorname{Ker} f_{x}$.

Proof. Let $\mu_{A}(x)=\alpha_{0}$ and $\nu_{A}(x)=\beta_{0}, \forall x \in \operatorname{Ker} f$, where $\alpha_{0}, \beta_{0} \in[0,1]$ are constants such that $\alpha_{0}+\beta_{0} \leq 1$. Let $g(x)=\sum_{i=0}^{m} b_{i} x^{i} \in \operatorname{Ker} f_{x}$, then

$0=f_{x}(g(x))=\sum_{i=0}^{m} f\left(b_{i}\right) x^{i} \Rightarrow f\left(b_{i}\right)=0, \forall i=1,2, \ldots, m$.

Hence $b_{i} \in \operatorname{Ker} f, \forall i=1,2, \ldots, m$, i.e., $\mu_{A}\left(b_{i}\right)=\alpha_{0}$ and $\nu_{A}\left(b_{i}\right)=\beta_{0} \forall i=1,2, \ldots, m$.

$\Rightarrow \mu_{A_{x}}(g(x))=\min _{i=0}^{m}\left\{\mu_{A}\left(b_{i}\right)\right\}=\alpha_{0}$ and $\nu_{A_{x}}(g(x))=\max _{i=0}^{m}\left\{\nu_{A}\left(b_{i}\right)\right\}=\beta_{0}$.

Hence $A_{x}$ is constant on $\operatorname{Ker} f_{x}$.

Corollary 3.10. Let $f: R \rightarrow R^{\prime}$ be an epimorphism from $R$ onto $R^{\prime}$ and let $f_{x}$ be the induced homomorphism of $f$. If an $f$-invariant intuitionistic fuzzy ideal $A$ of $R$ is constant on $\operatorname{Ker} f$, then $f_{x}\left(\sqrt{A_{x}}\right)=\sqrt{(f(A))_{x}}$. 
Proof. It follows from Proposition 3.8 and Theorem 3.5 that $f_{x}\left(\sqrt{A_{x}}\right)=\sqrt{f_{x}\left(A_{x}\right)}=\sqrt{(f(A))_{x}}$.

Definition 3.11. Let $A$ be an intuitionistic fuzzy ideal of a ring $R$. Then the intuitionistic fuzzy ideal $P(A)$ defined by

$$
P(A)=\cap\{B \mid A \subseteq B \text {, where } B \text { is an intuitionistic fuzzy prime ideal of } R\}
$$

is called an intuitionistic fuzzy prime radical of $A$.

Theorem 3.12. Let $A$ be an intuitionistic fuzzy ideal of a ring $R$ and let $A_{x}$ be its intuitionistic fuzzy polynomial ideal of $R[x]$. Then $P\left(A_{x}\right) \subseteq(P(A))_{x}$.

Proof. By Theorem 2.16, $B_{i}$ is an intuitionistic fuzzy ideal of $R$ with $A \subseteq B_{i}$ if and only if $\left(B_{i}\right)_{x}$ is an intuitionistic fuzzy prime ideal of $R[x]$ with $A_{x} \subseteq\left(B_{i}\right)_{x}$. It follows from Theorem 2.14 (i) that

$$
\begin{aligned}
(P(A))_{x} & =\left(\cap\left\{B_{i} \mid A \subseteq B_{i}, \text { where } B_{i} \text { is an intuitionistic fuzzy prime ideal of } R\right\}\right)_{x} \\
& =\left(\cap\left\{\left(B_{i}\right)_{x} \mid A \subseteq B_{i}, \text { where } B_{i} \text { is an intuitionistic fuzzy prime ideal of } R\right\}\right) \\
& \left.\subseteq \cap\left\{\left(B_{i}\right)_{x} \mid A_{x} \subseteq\left(B_{i}\right)_{x}, \text { where }\left(B_{i}\right)_{x} \text { is an intuitionistic fuzzy prime ideal of } R[x]\right\}\right) \\
& =\cap\left\{C_{i} \mid A_{x} \subseteq C_{i}, \text { where } C_{i} \text { is an intuitionistic fuzzy prime ideal of } R[x]\right\} \\
& =P\left(A_{x}\right) .
\end{aligned}
$$

This proves the theorem.

Remark 3.13. Let $A$ be an intuitionistic fuzzy ideal of a ring $R$ and let $A_{x}$ be its intuitionistic fuzzy polynomial ideal of $R[x]$. We denote

$\operatorname{IFPI}(A)=\{B \mid A \subseteq B$, where $B$ is an intuitionistic fuzzy prime ideal of $R\}$, and $\operatorname{IFPI}\left(A_{x}\right)=\left\{D \mid A_{x} \subseteq D\right.$, where $D$ is an intuitionistic fuzzy prime ideal of $\left.R[x]\right\}$.

Theorem 3.14. Let $A$ be an intuitionistic fuzzy ideal of a ring $R$ and let $A_{x}$ be its intuitionistic fuzzy polynomial ideal of $R[x]$. Then the map

$$
\phi: \operatorname{IFP} A(A) \rightarrow \operatorname{IFPI}\left(A_{x}\right) \text { defined by } \phi(B)=B_{x},
$$

is one-one.

Proof. Let $B, C \in \operatorname{IFPI}(A)$ such that $\phi(B)=\phi(C)$, then $B_{x}=C_{x}$. It follows that $\left(B_{x}\right)(r)=$ $\left(C_{x}\right)(r)$, for all $r \in R$, and hence $B(r)=C(r)$ for all $r \in R$, proving that $B=C$. Hence $\phi$ is one-one.

Corollary 3.15. Let $A$ be an intuitionistic fuzzy ideal of a ring $R$ and let $A_{x}$ be its intuitionistic fuzzy polynomial ideal of $R[x]$. If the map $\phi$ defined in Theorem 3.14 is one-one map, then $(P(A))_{x}=P\left(A_{x}\right)$.

Proof. If $D$ is any element of $I F P I\left(A_{x}\right)$, then there exists $C \in I F P I(A)$ with $A \subseteq C$ such that $C_{x}=\phi(C)=D$. Thus $(P(A))_{x}=P\left(A_{x}\right)$. 
Example 3.16. Let $\mathbf{Z}$ be the set of all integers. Define an IFS $A$ on $\mathbf{Z}$ by

$$
\mu_{A}(x)=\left\{\begin{array}{ll}
1, & \text { if } x \in 2 \mathbf{Z} \\
0, & \text { otherwise }
\end{array} ; \quad \nu_{A}(x)=\left\{\begin{array}{ll}
0, & \text { if } x \in 2 \mathbf{Z} \\
1, & \text { otherwise }
\end{array} .\right.\right.
$$

Then $A$ is an intuitionistic fuzzy prime ideal of $\mathbf{Z}$, for $A_{*}=2 \mathbf{Z}$ is a prime ideal of $\mathbf{Z}$, and its induced polynomial ideal $A_{x}$ is given by

$$
\mu_{A_{x}}(f(x))=\left\{\begin{array}{ll}
1, & \text { if } f(x) \in 2 \mathbf{Z}[\mathbf{x}] \\
0, & \text { otherwise }
\end{array} ; \quad \nu_{A_{x}}(f(x))= \begin{cases}0, & \text { if } f(x) \in 2 \mathbf{Z}[\mathbf{x}] \\
1, & \text { otherwise }\end{cases}\right.
$$

By Theorem 2.16, the intuitionistic fuzzy polynomial ideal $A_{x}$ induced by $A$ is an intuitionistic fuzzy polynomial ideal of $\mathbf{Z}[\mathbf{x}]$. Hence $(P(A))_{x}=A_{x}=P\left(A_{x}\right)$.

\section{Acknowledgements}

The second author would like to thank IKG PT University, Jalandhar for providing the opportunity to do research work.

\section{References}

[1] Atanassov, K. T. (1986). Intuitionistic fuzzy sets, Fuzzy Sets and Systems, 20(1) 87-96.

[2] Atanassov, K. T. (1999). Intuitionistic Fuzzy Sets: Theory and Applications, Studies on Fuzziness and Soft Computing, 35, Physica-Verlag, Heidelberg.

[3] Bakhadach, I., Melliani, S., Oukessou, M., \& Chadli, L. S. (2016). Intuitionistic fuzzy ideal and intuitionistic fuzzy prime ideal in a ring, Notes on Intuitionistic Fuzzy Sets, 22(2), 59-63.

[4] Banerjee, B., \& Basnet, D. K. (2003). Intuitionistic fuzzy subrings and ideals, The Journal of Fuzzy Mathematics, 11(1), 139-155.

[5] Biswas, R. (1989). Intuitionistic fuzzy subgroup, Mathematical Forum, X, 37-46.

[6] Hur, K., Jang, S. Y., \& Kang, H. W. (2005). Intuitionistic Fuzzy Ideals of a Ring, Journal of the Korea Society of Mathematical Education, Series B, 12(3), 193-209.

[7] Hur, K., Kang, H. W., \& Song, H. K. (2003). Intuitionistic Fuzzy Subgroups and Subrings, Honam Math J., 25(1), 19-41.

[8] Jun, Y. B., Ozturk, M. A., \& Park, C. H. (2007). Intuitionistic nil radicals of intuitionistic fuzzy ideals and Euclidian intuitionistif fuzzy ideals in rings, Information Sciences, 177, $4662-4677$. 
[9] Kim, C. B., Kim, H. K., \& So, K. S. (2014). On the fuzzy polynimial ideals, Journal of Intelligent and Fuzzy Systems, 27, 487-494.

[10] Meena, K. (2017). Characteristic intuitionistic fuzzy subrings of an intuitionistic fuzzy ring, Advances in Fuzzy Mathematics, 12(2), 229-253.

[11] Meena, K., \& Thomas, K. V. (2011). Intuitionistic L-fuzzy Subrings, International Mathematical Forum, 6(52), 2561-2572.

[12] Sharma, P. K. (2011). Translates of intuitionistic fuzzy subring, International Review of Fuzzy Mathematics, 6(2), 77-84.

[13] Sharma, P. K., \& Kaur, G. (2018). On the intuitionistic fuzzy polynomial ideals, Notes on Intuitionistic Fuzzy Sets, 24(1), 48-59.

[14] Zadeh, L. A. (1965). Fuzzy sets, Information and Control, 8, 338-353. 